\title{
Comparison of Two Experiments Based on a Physical and a Torsion Pendulum to Determine the Mass Moment of Inertia Including Measurement Uncertainties
}

\author{
Leonard Klaus \\ Physikalisch-Technische Bundesanstalt(PTB), Bundesallee 100, 38116 Braunschweig, Germany, leonard.klaus@ptb.de
}

\begin{abstract}
To determine the mass-moment-of-inertia properties of devices under test with particularly small mass moments of inertia $\left(\right.$ some $\left.10^{-4} \mathrm{~kg} \mathrm{~m}^{2}\right)$, two measurement set-ups based on different measurement principles were developed. One set-up is based on a physical pendulum, the second set-up incorporates a torsion pendulum. Both measurement set-ups and their measurement principles are described in detail, including the chosen data acquisition and analysis. Measurement uncertainty estimations according to the Guide to the Expression of Uncertainty in Measurement (GUM) were carried out for both set-ups by applying Monte Carlo simulations. Both set-ups were compared using the same three devices under test. For each measurement result, the measurement uncertainties were estimated. The measurement results are compared in terms of consistency and the resulting measurement uncertainties. For the given devices under test, the torsion pendulum set-up gave results with smaller measurement uncertainties compared to the set-up incorporating a physical pendulum.
\end{abstract}

Keywords: Torsional pendulum, calibration, measurement uncertainty evaluation.

\section{INTRODUCTION}

Determining the mass moment of inertia (MMOI) precisely can be important in many applications. For simpler mechanical designs, a calculation based on technical drawings might be sufficient. A measurement is required in the case of a complex design, various materials or if assigned measurement uncertainties are needed.

\subsection{Measurement principles and realised set-ups}

The experimental determination of the mass moment of inertia based on pendulums is a well-tried method. It is based on the measurement of the pendulum swing frequency, which is dependent on the mass moment of inertia of the device under test (DUT). Approaches based on torsional oscillations feature linear equations of motion and can be implemented as torsion pendulums or filar pendulums [1]. Bi-filar to multi-filar pendulum approaches have been used from the 1930s [2,3] up to now [4] for the measurement of the mass moment of inertia, especially for heavy objects. The advantage of these approaches is that only few modifications to the device under test are necessary, the set-up is simple, and the added oscillating components have a very small additional MMOI. However, the precision is limited due to the imprecise measurement of the swing frequency and motion in additional degrees of freedom. Both disadvantages may be overcome with more advanced set-ups which cancel out the above-mentioned advantages to some extent due to the higher complexity [5]. Torsion pendu- lum set-ups are typically used for DUTs with smaller mass moments of inertia [6].

Physical pendulums have also been used for the determination of the mass moment of inertia to a lesser extent [7, 8] or in conjunction with a filar pendulum for measurements in multiple axes $[2,3]$.

\subsection{Purpose of the mass moment of inertia measurements}

The combination of the aforementioned requirements for the experimental determination of the mass moment of inertia was the motivation for the design of the set-ups described in this contribution. For the development of a model-based approach for the dynamic calibration of torque transducers [9], different components of a corresponding measurement device had to be analysed in terms of their mechanical properties; amongst other things in terms of the mass moment of inertia. The devices under test have complex mechanical designs, are consisting of different materials and - most importantly - the measurement uncertainties need to be assigned to the measurement results. To this end, two set-ups based on a physical pendulum and on a torsion pendulum were designed, manufactured, and commissioned.

Compared to the presented previously realised set-ups, the specimens for which the mass moment of inertia had to be determined are comparably small and have a low mass moment of inertia in the range of some $10^{-4} \mathrm{~kg} \mathrm{~m}^{2}$ rising unique design issues for the set-ups. Typical examples of devices under test 


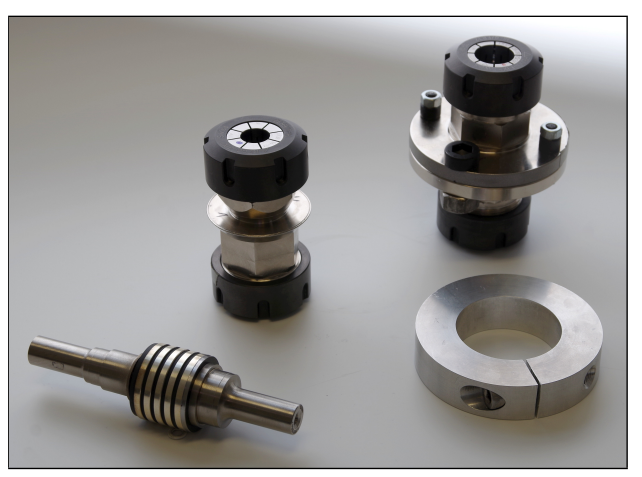

Fig.1. Typical specimens for which the mass moment of inertia had to be determined by means of the presented pendulum set-ups.

are shown in Figure 1. A first determination of some massmoment-of-inertia properties of the dynamic torque calibration device was already carried out in 2006 for one part of the calibration device. The chosen method of a torsion pendulum showed unsatisfactory non-linear effects. Aside from that, the results - including measurement accuracies - were good [10]

In the following sections, both set-ups will be described and compared.

\section{PHYSICAL PENDULUM}

Additional measurements became necessary after modifications to the measuring device and for the development of a model-based calibration approach. To this end, a new set-up based on a physical pendulum was designed. A short overview can be found in [11].

\subsection{Measurement principle}

A physical pendulum oscillates around a horizontal axis of rotation after being displaced from its vertical equilibrium position, as depicted in Figure 2.

The (ideal) undamped motion of the pendulum is described by

$$
J \cdot \ddot{\vartheta}(t)+m \cdot g_{\text {loc }} \cdot l \cdot \sin \vartheta(t)=0
$$

with the pendulum's mass moment of inertia $J$, the excitation angle $\vartheta$, the mass of the pendulum $m$, the distance of the pendulum's centre of gravity $s$ to its axis of rotation $l$ and the local gravitational acceleration $g_{\text {loc }}$. For small angles of excitation, the latter equation can be linearised applying the small-angle approximation $\sin \varphi \approx \varphi$ giving

$$
J \cdot \ddot{\vartheta}(t)+m \cdot g_{\mathrm{loc}} \cdot l \cdot \vartheta(t)=0
$$

In the frequency domain, the relation of the mass moment of inertia and the squared angular pendulum swing frequency $\omega^{2}$ of the linearised equation becomes obvious giving

$$
J=\frac{m \cdot g_{\mathrm{loc}} \cdot l}{\omega^{2}}
$$

The simplifying assumption of linear and undamped oscillations causes deviations, which have to be taken into account for the measurement uncertainty evaluation.

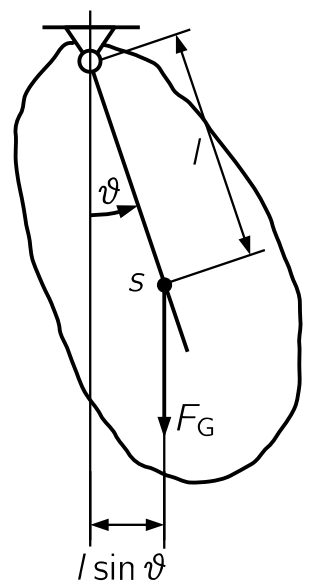

Fig. 2. Schematic diagram of a physical pendulum.

\subsection{Realised set-up}

To realise oscillations with very low damping, the bearing of the pendulum was realised using a spherical air bearing with an aluminium pendulum lever (as depicted in Figure 3). The friction of the bearing was previously analysed for other purposes [12]. Although the friction of the chosen bearing was comparably high compared to other air bearings, it was assumed to be sufficiently low for the given application in conjunction with being robust against bending moments.

On one side of the air bearing, the pendulum lever was mounted by using a centring mechanism, positioning it at a defined position. Mounted on the other side of the air bearing, a precise angle measurement system consisting of a radial grating disk and an incremental scanning head provides time series data of the pendulum excitation angle with high resolution. The analogue quadrature voltage output signals are interpolated and converted to digital TTL signals, before being acquired and analysed using a counter/timer data acquisition card.

With the bearing and the mounted additional components, the pendulum itself has an unknown mass moment of inertia, which is denoted as $J_{0}$. To be able to carry out measurements, a known detuning of the pendulum is required for an identification of $J_{0}$, or in the case of a measurement with a mounted device under test: $J_{0}+J_{\text {DUT }}$. The detuning was realised by adding mass bodies at different distances from the axis of rotation at well-defined positions. For this purpose, the pendulum lever was designed with mounting holes. Each mass body has a cylindrical shape with known dimensions and mass (cf. Figure 7). The mass moment of inertia of each mass body around its cylinder axis can be calculated giving

$$
J_{\mathrm{cyl}}=\frac{m \cdot r^{2}}{2}
$$

with the mass $m$ and the radius $r$. The Huygens-Steiner theorem gives the acting mass moment of inertia for a mass body at its mounted position at a distance $l$ from the axis of rotation

$$
J_{\text {shifted }}=J_{\text {cyl }}+m \cdot l^{2} .
$$




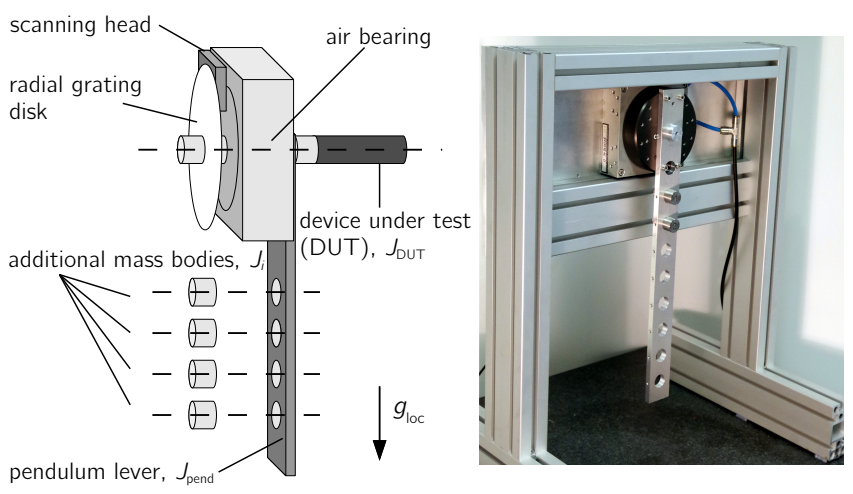

Fig.3. Sketch of the physical pendulum set-up (left) and photograph of the set-up including two added mass bodies mounted on the pendulum lever (right).

The mounting position is known due to measurements of the lever dimensions by means of a coordinate measurement system. However, not only the term of $J$ in Equation (3) will be affected by the added mass bodies, but also the mass of the pendulum and the position of the centre of gravity as well.

The measurement of the mass moment of inertia is carried out with different configurations of added mass bodies, giving for each configuration $i$ the detuned pendulum's equation (cf. (3))

$$
J_{0}+J_{\mathrm{DUT}}+\sum J_{i}=\frac{m_{0} \cdot g_{\mathrm{loc}} \cdot l_{0}+\sum m_{i} \cdot g_{\mathrm{loc}} \cdot l_{i}}{\omega_{i}^{2}}
$$

The devices under test will not contribute to the restoring torque, because they will be mounted on the axis of rotation. Due to the known $J_{i}, m_{i}$, and $l_{i}$, an approximation of the unknown $J_{0}+J_{\text {DUT }}$ and restoring torque $m_{0} \cdot g_{\text {loc }} \cdot l_{0}$ is possible. The higher the number of measurement configurations with different mass bodies, the lower the uncertainty of the estimate.

\subsection{Data analysis}

The identification of the mass moment of inertia was carried out using a number of measurements with different numbers of mass bodies at different positions in the pendulum lever. The swing frequency of each measurement was determined applying a sine fit algorithm to the time series data set. To this end, a damped sine function

$$
y(t)=\hat{y}_{0} \cdot \mathrm{e}^{-\delta t} \cdot \sin (\omega t+\varphi)+B
$$

with the magnitude $\hat{y}_{0}$, offset $B$, decay rate $\delta$, angular frequency $\omega$, and phase angle $\varphi$ was approximated to the measurement data. The procedure of the data acquisition and analysis is diagrammed in Figure 4.

The parameter approximation to derive the unknown mass moment of inertia and the restoring torque is carried out as a bivariate regression incorporating a non-linear model function G

$$
y=G(X, \theta)
$$

relating the measured frequencies $\omega_{i}$ in the vector of observed

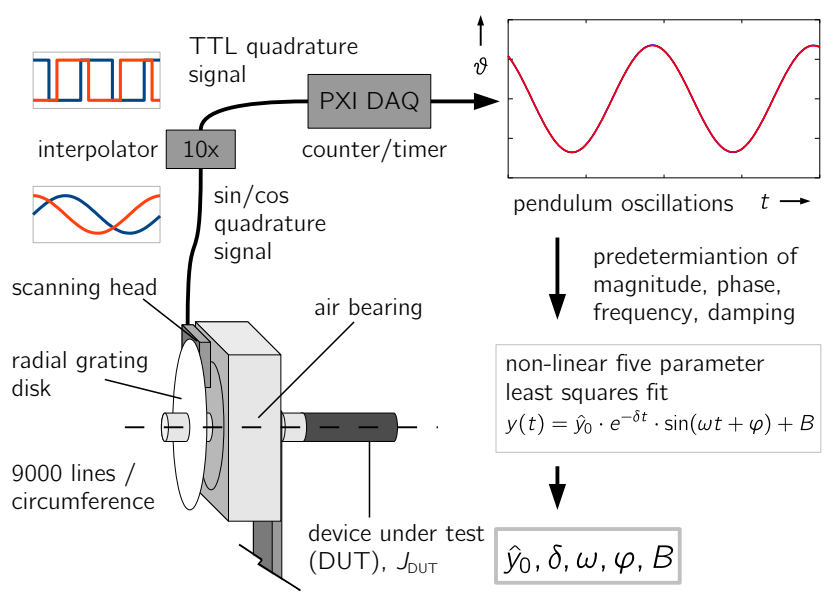

Fig.4. Angle measurement, data acquisition and processing for the physical pendulum set-up.

values $y$

$$
y=\left[\omega_{1}, \omega_{2}, \ldots, \omega_{n}\right]^{\mathrm{T}}
$$

to the matrix of independent values $X$ consisting of the added mass moments of inertia and the restoring torque values of the corresponding configurations

$$
X=\left[\begin{array}{cc}
\sum J_{1} & \sum\left(m_{1} \cdot g_{\text {loc }} \cdot l_{1}\right) \\
\sum J_{2} & \sum\left(m_{2} \cdot g_{\text {loc }} \cdot l_{2}\right) \\
\ldots & \ldots \\
\sum J_{n} & \sum\left(m_{n} \cdot g_{\text {loc }} \cdot l_{n}\right)
\end{array}\right],
$$

and also to the vector of approximated parameters $\theta$ as

$$
\theta=\left[J_{0}+J_{\mathrm{DUT}}, \sum\left(m_{0} \cdot g_{\mathrm{loc}} \cdot l_{0}\right)\right]^{\mathrm{T}} .
$$

For the parameter estimation, an iterative least squares estimator was applied to the non-linear model equation. The sum of the squared errors is minimised in order to estimate the parameters giving

$$
\hat{\theta}=\arg \min _{\hat{\theta}}(y-G(X, \theta))^{2} .
$$

For the determination of the mass moment of inertia of a DUT, in a first step the properties of the pendulum itself $\left(J_{0}, m_{0}\right.$. $g_{\text {loc }} \cdot l_{0}$ ) had to be determined. Consecutively, it was possible to carry out additional measurements with the mounted DUT. The mass moment of inertia was derived by the subtraction of the two measurement results as follows

$$
J_{\text {DUT }}=\left(J_{0}+J_{\text {DUT }}\right)-J_{0} .
$$

\subsection{Deviations due to linearisation}

The identification of the mass moment of inertia is based on the assumption of a linear behaviour of the physical pendulum. The real pendulum's swing time is dependent on the pendulum excitation angle, and will only converge to the linear behaviour for small pendulum excitation angles. For the measurement uncertainty evaluation, the influence of the non-linearity had to be estimated. The pendulum equation (cf. (1)) - although 


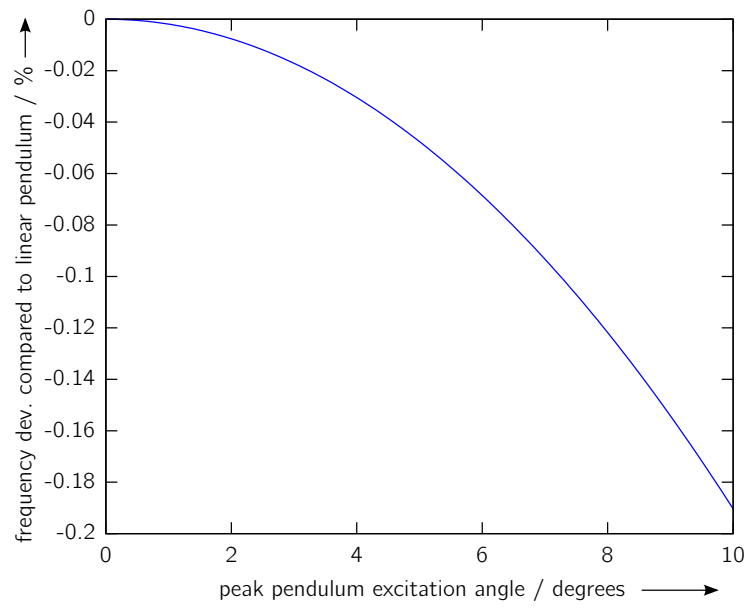

Fig.5. Deviation of the physical pendulum swing frequency and a linear model assumption as a function of the peak excitation angle.

apparently simple - is not easy to solve and was investigated in several publications $[7,13,14,15]$. The deviations due to the linearisation were quantised with regard to the peak excitation angle $\vartheta_{\text {s }}$ of the pendulum.

The pendulum swing time $\tau_{\vartheta_{\mathrm{s}}}$ of the non-linear physical pendulum is given [7] as

$$
\tau_{\vartheta_{\mathrm{s}}}=\frac{2}{\pi} \tau_{0} K(k)
$$

with the swing time $\tau_{0}$ of the linear solution (cf. Equation (2))

$$
\tau_{0}=2 \pi \sqrt{\frac{J}{m \cdot l \cdot g_{\mathrm{loc}}}},
$$

and the elliptic integral of the first kind $K(k)$ [16, pp. 569]

$$
K(k)=\int_{0}^{\frac{\pi}{2}} \frac{\mathrm{d} \varphi}{\left(1-k^{2} \sin ^{2} \varphi\right)^{\frac{1}{2}}} .
$$

For the non-linear pendulum with the excitation angle $\vartheta_{\mathrm{s}}$, the expression below follows for $k$ [17, pp. 208]

$$
k=\sin \left(\frac{\vartheta_{\mathrm{s}}}{2}\right)
$$

Therefore, the ratio of linear and non-linear pendulum swing time is given as

$$
\frac{\tau_{\vartheta_{\mathrm{s}}}}{\tau_{0}}=\frac{2}{\pi} \int_{0}^{\frac{\pi}{2}} \frac{\mathrm{d} \varphi}{\left(1-k^{2} \sin ^{2} \varphi\right)^{\frac{1}{2}}} .
$$

Figure 5 depicts the calculated deviations of the pendulum swing frequencies of the linear and non-linear model. It becomes obvious that the deviations are very small for small pendulum excitation angles. The remaining influences of the chosen peak excitation angles of less than $2.5^{\circ}$ are only in a range of $1 \times 10^{-4}$ and are taken into account for the measurement uncertainty estimation.

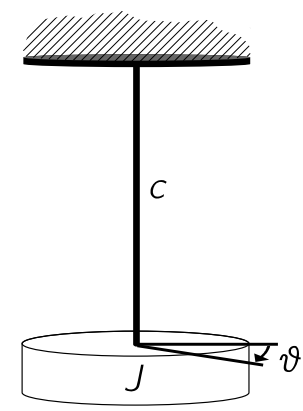

Fig.6. Schematic diagram of a torsion pendulum with the spring $c$ and mass moment of inertia $J$.

\section{TORSION PENDULUM}

A second approach implemented for the determination of the mass moment of inertia was a torsion pendulum.

\subsection{Measurement principle}

A torsion pendulum consists of typically vertically arranged components which are able to oscillate rotationally. Differently from a physical pendulum, the energy of the pendulum is stored in a torsion spring. This spring is fixed at one end, while the other end can rotate freely. A schematic diagram of a torsion pendulum is depicted in Figure 6. After an excitation, oscillations with a natural frequency related to the acting mass moment of inertia will occur.

The homogeneous equation of motion under the assumption of undamped oscillations follows as

$$
J \cdot \ddot{\vartheta}(t)+c \cdot \vartheta(t)=0
$$

with the angle of excitation $\vartheta$, the torsional stiffness of the spring $c$, and the mass moment of inertia $J$. In the frequency domain, the linear relation of the inverse of the squared angular swing frequency $\omega$ and the mass moment of inertia becomes obvious

$$
J=\frac{c}{\omega^{2}} .
$$

Due to the fact that the torsional stiffness of the spring used in a set-up for the determination of the mass moment of inertia is usually unknown, an approach comparable to the one chosen for the physical pendulum had to be applied. Multiple measurements with different added known mass bodies were used to estimate the mass moment of inertia of a device under test by applying a linear regression. The mass-moment-of-inertia elements used were made of brass and are shown in Figure 7.

\subsection{Measurement set-up}

The measurement set-up of the torsion pendulum is based on components of the dynamic torque calibration device, whose mass-moment-of-inertia properties had to be determined. The set-up is depicted in Figure 8. This approach has numerous advantages over a dedicated measurement set-up: 


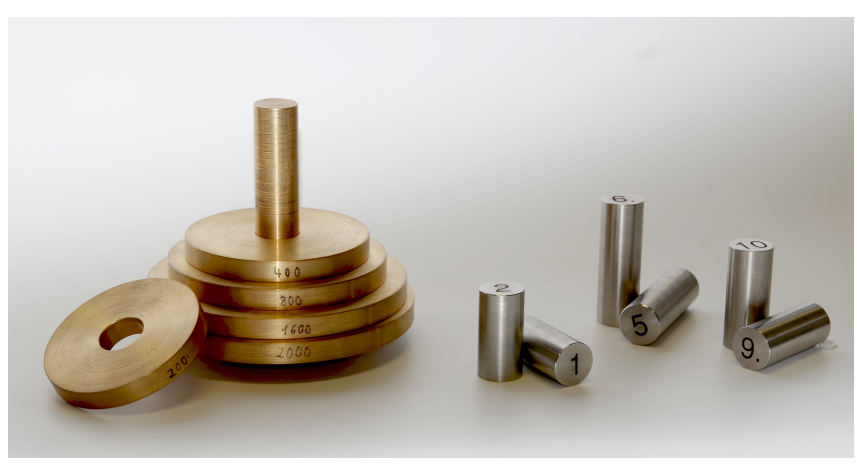

Fig.7. Added mass-moment-of-inertia elements for the torsion pendulum (left) and for the physical pendulum (right).

- Only small modifications to the dynamic torque calibration device are needed for the measurement of the mass moment of inertia

- Measurements are performed under the same conditions as in the later dynamic torque calibration application

- Angle measurement components are already existing and adjusted

- Set-up can be established in a short time.

As already mentioned, a similar approach designed and commissioned a decade ago showed non-linear behaviour. The source of the non-linearities was not clearly identified. Several actions were taken to minimise non-linearities in the actual set-up:

- Press-fitted upper and lower mounting parts with large overlap areas to the spring to reduce local stress maxima

- Longer torsion spring to limit strain despite a larger diameter of the spring compared to the first set-up

- Small angles of excitation to reduce strain.

The integration of the torsion pendulum in the dynamic torque calibration device was carried out so that the pendulum was detachable. The torsion spring is mounted on the top of the rotor of an air bearing, and a radial grating disk is mounted below. An incremental scanning head measures the pendulum excitation angle utilising the grating disk. The additional mass bodies are mounted using a coupling element.

To avoid any modifications to the rotating components of the dynamic torque calibration device, the lower part of the torsion spring needed to be connected without any permanent mounting components. Therefore, a disk-shaped lower ending of the torsion spring was designed, which was connected to the air bearing's rotor by means of bonding wax. The applied Stronghold Blanchard Wax 7036 - typically used for the temporary mounting of semiconductor wafers - is recommended for the mounting of accelerometers [18]. Being solid at room temperature, the wax starts to soften if heated to temperatures above $70^{\circ} \mathrm{C}$. It can be removed easily using acetone as a solvent.

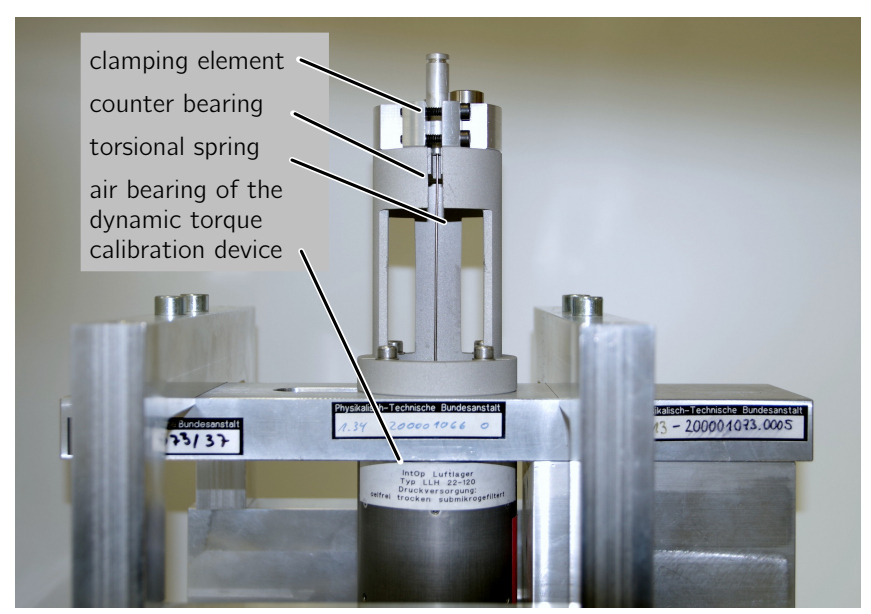

Fig. 8. Set-up for the determination of the mass moment of inertia based on a torsion pendulum.

For mounting the torsion spring to the rotor of the air bearing, both parts were heated to temperatures high enough to make the mounting wax completely liquid. Both components were pressed together to obtain a thin and stable layer of wax.

\subsection{Data analysis}

The measurement of the time-varying angle excitation of the torsion pendulum was carried out similarly to the physical pendulum by means of an incremental angle measurement system, and a sine function was applied to the time series data (cf. Equation (7)). After deriving the swing frequencies, the actual MMOI of the device under test was determined by means of a linear regression. From the inverse of Equation (20) the expression below follows for the squared pendulum swing time

$$
\tau^{2}=\frac{4 \pi^{2}}{c}\left(J_{\mathrm{DUT}}+J_{i}\right) .
$$

The line equation has a zero at $J_{i}=-J_{\text {DUT }}$. For a number of measurements with different known added mass-moment-ofinertia bodies $J_{i}$, a line equation can be approximated to the measurement data. The intersection of the approximated line and the $\mathrm{x}$-axis gives the extrapolated mass moment of inertia of the DUT, as depicted in Figure 9.

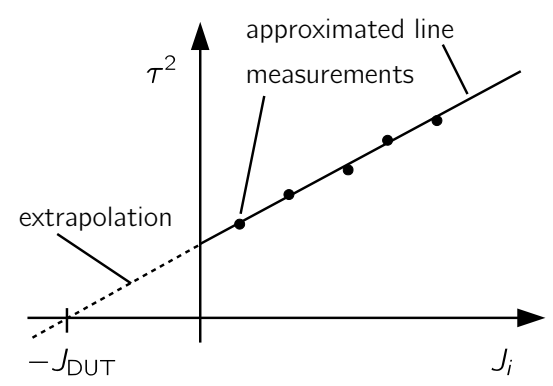

Fig.9. Determination of the mass moment of inertia by means of a line approximation. 


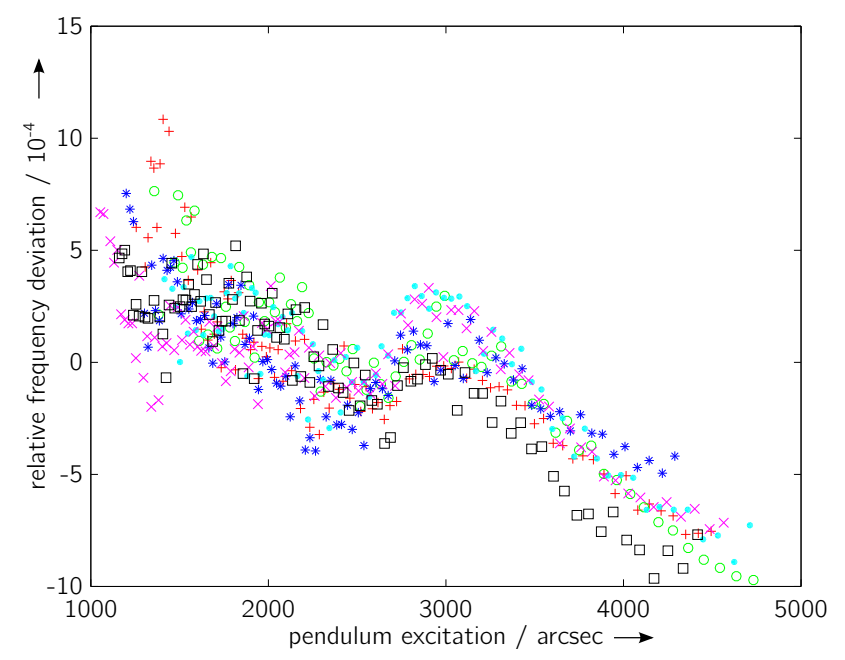

Fig. 10. Dependency of the relative deviation of the pendulum swing frequency on the pendulum excitation angle for each mass body configuration.

The mass moment of inertia of the pendulum itself, i.e. of the top part of the measuring device, needs to be subtracted, depending on for which device under test the mass moment of inertia is to be determined. This is similar to the procedure applied for the physical pendulum set-up (cf. Equation (13)).

\subsection{Non-linearities}

The linearity of the torsion pendulum is one advantage if choosing this design. However, the set-up which was first established experienced significant non-linear behaviour. As mentioned before, the design of the new torsion pendulum set-up was optimised in order to minimise non-linearity. The non-linear behaviour becomes obvious if there is an excitationdependent change in the pendulum swing frequency.

After commissioning the new pendulum set-up, dedicated analyses were carried out to quantise the amount of non-linear behaviour. For this purpose, time series data of pendulum swings was divided into segments of about one oscillation. The frequency of each oscillation was determined by means of a sine fit. The results are depicted as the swing-frequency deviation versus excitation in Figure 10.

It becomes obvious from the results that small non-linear effects still remain. The swing frequency clearly increases with decreasing swing amplitude. This behaviour is similar for all measurements and is most likely caused by the mounting of the torsion spring with wax. The rotor of the air bearing was modified in the set-up which was first established in order to mount the torsion spring. This was not possible in the latter case.

The magnitude of the pendulum frequency change was significantly reduced by a factor of 10 . While the first set-up experienced a frequency change of about $1 \%$ relative to the mean value per an excitation of 1000 arcsec, this non-linear effect was reduced to $0.1 \%$ in the actual set-up.

\section{Measurement unCERTAinty EVAluation}

The measurement uncertainty of both set-ups was estimated based on the Guide to the Expression of Uncertainty in Measurement (GUM) [19] and its Supplement 1 (GUM S1) [20], respectively. The evaluation of the measurement uncertainty according to the GUM is based on a mathematical model of the measurement itself. Measurement uncertainty contributions due to different input quantities $X_{1}, X_{2}, \ldots, X_{n}$ are propagated to the measurement result $Y$ based on the measurement's model function giving

$$
Y=f\left(X_{1}, X_{2}, \ldots, X_{n}\right) \text {. }
$$

Every single input quantity is characterised by its probability density function (PDF), leading to a corresponding PDF of the measurement result.

The classic estimation of measurement uncertainties in the GUM based on partial derivative calculations is limited in terms of possible input PDFs and assumes a linear measurement model. The approach proposed in the GUM S1 incorporates a repeated simulation of the measurement process by means of Monte Carlo simulations. The measurement uncertainties of the different input quantities are simulated by their PDFs. Each simulation is carried out with random samples drawn from the PDFs.

\subsection{Dependency of estimated uncertainty and number of Monte Carlo runs}

The outcome of Monte Carlo simulations may be dependent on the number of simulation runs. If the number of runs is too low, the estimated uncertainties may be too high or too low. To avoid this problem, a sufficiently high number of simulations have to be carried out. Unfortunately, this number is dependent on the application and therefore cannot be known in advance. To overcome this problem, different proposals to define a stopping criterion have been analysed [21]. For the given application, the uncertainty calculation was carried out for different numbers of Monte Carlo runs. After analysing the results, a significantly high number of runs (conservative safety margin added) were chosen.

\subsection{Damping}

The models for the determination of the mass moment of inertia for both measurement set-ups assume undamped oscillations. Although the damping in both set-ups was minimised by all measures (mechanical design, use of air bearings), there still might be influences which have to be taken into account for the measurement uncertainty evaluation.

The influence of the remaining damping was estimated by analysing the decay of the pendulum swing over time. To this end, the pendulum oscillations were acquired and a damped sine function (cf. Equation (7)) was approximated to the acquired time series data. The parameters of interest are, in this case, the decay rate $\delta$ and the pendulum's damped angular frequency $\omega_{\mathrm{d}}$. Both relate to the (theoretical) undamped pend- 
Table 1. Uncertainty contributions for the determination of the physical pendulum set-up.

\begin{tabular}{|c|c|c|}
\hline input quantity & $\begin{array}{l}\text { uncertainty } \\
\text { contribution }\end{array}$ & $\begin{array}{l}\text { distribution/ } \\
\text { correlated }(\mathrm{y} / \mathrm{n})\end{array}$ \\
\hline $\begin{array}{l}\text { angle deviation grating } \\
\text { angle dev. signal period } \\
\text { angle dev. eccentricity }\end{array}$ & $\begin{array}{l}u=0.033 \operatorname{arcsec} \\
u=1.44 \operatorname{arcsec} \\
u=0.39 \operatorname{arcsec}\end{array}$ & $\begin{array}{l}\text { Gaussian }(n) \\
\text { rectangular }(n) \\
\text { rectangular }(n)\end{array}$ \\
\hline $\begin{array}{l}\text { frequency deviation } \\
\text { non-linearity }\end{array}$ & $u_{\mathrm{rel}}=1 \times 10^{-4}$ & Gaussian (y) \\
\hline $\begin{array}{l}\text { frequency deviation } \\
\text { damping }\end{array}$ & $u_{\mathrm{rel}}=1 \times 10^{-7}$ & Gaussian (y) \\
\hline oscillator PXI system & $u_{\text {rel }}=2.5 \times 10^{-6}$ & rectangular (n) \\
\hline $\begin{array}{l}\text { local gravitational } \\
\text { acceleration } g_{\text {loc }}\end{array}$ & $u_{\mathrm{rel}}=5 \times 10^{-8}$ & Gaussian (n) \\
\hline $\begin{array}{l}\text { pendulum tilt } \\
\text { determination of mass } \\
\text { pendulum position } \\
\text { dimensions mass bodies } \\
\text { bevels of mass bodies }\end{array}$ & $\begin{array}{l}u_{\text {rel }}=1.25 \times 10^{-7} \\
u=5 \times 10^{-8} \mathrm{~kg} \\
u=2 \times 10^{-6} \mathrm{~m} \\
u=2 \times 10^{-6} \mathrm{~m} \\
u=4 \times 10^{-9} \mathrm{~kg} \mathrm{~m}^{2}\end{array}$ & $\begin{array}{l}\text { Gaussian (y) } \\
\text { Gaussian (y) } \\
\text { Gaussian (n) } \\
\text { Gaussian (y) } \\
\text { Gaussian (y) }\end{array}$ \\
\hline
\end{tabular}

Table 2. Uncertainty contributions for the determination of the torsion pendulum set-up.

\begin{tabular}{llc}
\hline input quantity & $\begin{array}{l}\text { uncertainty } \\
\text { contribution }\end{array}$ & distribution \\
\hline $\begin{array}{l}\text { angle deviation grating } \\
\text { angle dev. signal period } \\
\text { angle dev. eccentricity } \\
\text { frequency deviation }\end{array}$ & $\begin{array}{l}u=0.06 \mathrm{arcsec} \\
\text { non-linearity }\end{array}$ & $\begin{array}{c}\text { Gaussian } \\
\text { rectangular } \\
\text { rectangular }\end{array}$ \\
$\begin{array}{l}\text { frequency deviation } \\
\text { damping }\end{array}$ & $u_{\mathrm{rel}}=1 \times 10^{-3}$ & Gaussian \\
$\begin{array}{l}\text { oscillator PXI system } \\
\text { mass bodies }\end{array}$ & $u_{\mathrm{rel}}=2.1 \times 10^{-6}$ & Gaussian \\
$J$ torsion spring & $u=9.48 \times 10^{-6} \mathrm{~kg} \mathrm{~m}^{2}$ & Gaussian \\
$J$ mounting disk & $u=2.2 \times 10^{-10} \mathrm{~kg} \mathrm{~m}^{2}$ & Gaussian \\
$J$ wax film & $u=7.9 \times 10^{-9} \mathrm{~kg} \mathrm{~m}^{2}$ & Gaussian \\
\hline
\end{tabular}

ulum oscillation frequency $\omega_{0}$ giving

$$
\omega_{0}=\sqrt{\delta^{2}+\omega_{\mathrm{d}}^{2}} .
$$

Data from several measurements derived values of $2.1 \times 10^{-6}$ for the torsion pendulum set-up and $1 \times 10^{-7}$ for the physical pendulum set-up for the maximum relative deviation due to the damping.

\subsection{Uncertainty contributions for the two set-ups}

For each of the set-ups, the different measurement uncertainty contributions were identified and quantised.

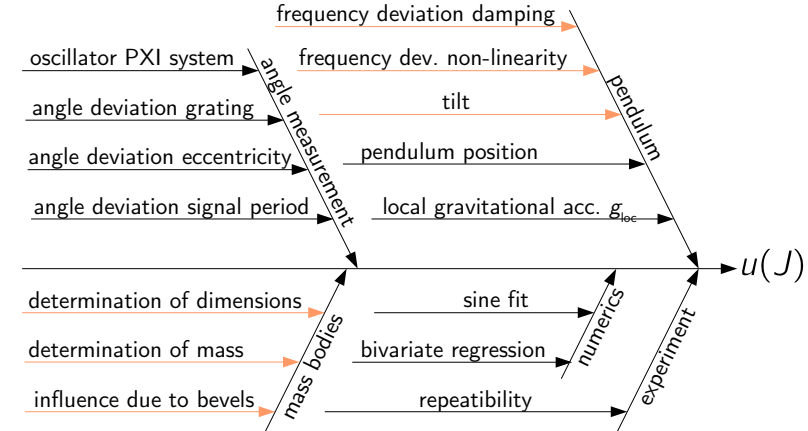

Fig. 11. Ishikawa diagram of the measurement uncertainty contributions of the set-up based on a physical pendulum. Correlated uncertainty contributions are marked in orange.

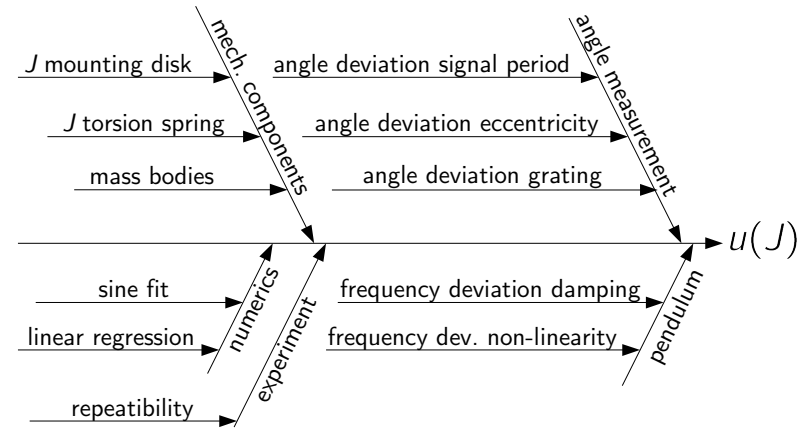

Fig.12. Ishikawa diagram of the measurement uncertainty contributions of the set-up based on a torsion pendulum.

The different uncertainty contributions of the physical pendulum are displayed in Figure 11 as an Ishikawa diagram, and are given in detail in Table 1. The same information for the torsion pendulum set-up can be found in Figure 12 and Table 2, respectively.

For each measurement, i.e. each set of different time series data files of pendulum measurements with varying added massmoment-of-inertia elements, Monte Carlo simulations were carried out in order to estimate the measurement uncertainty. The calculations were performed using the open source numerical computation software GNU Octave [22]. The Monte Carlo simulations included the whole data analysis applying uncertainty contributions of the time series data to the measurement data files, uncertainty contributions corresponding to fit parameters in the corresponding code for the parameter estimation, and so on.

The physical pendulum set-up contains correlated uncertainty contributions (marked orange in Figure 11) due to the subtraction of the mass moment of inertia of the pendulum itself $\left(J_{0}\right)$, which was derived by a second measurement. These correlated uncertainty contributions were applied by sampling from the same random number generator. Therefore, the two measurements of $J_{0}+J_{\text {DUT }}$ and $J_{0}$ were included in one Monte Carlo simulation. 


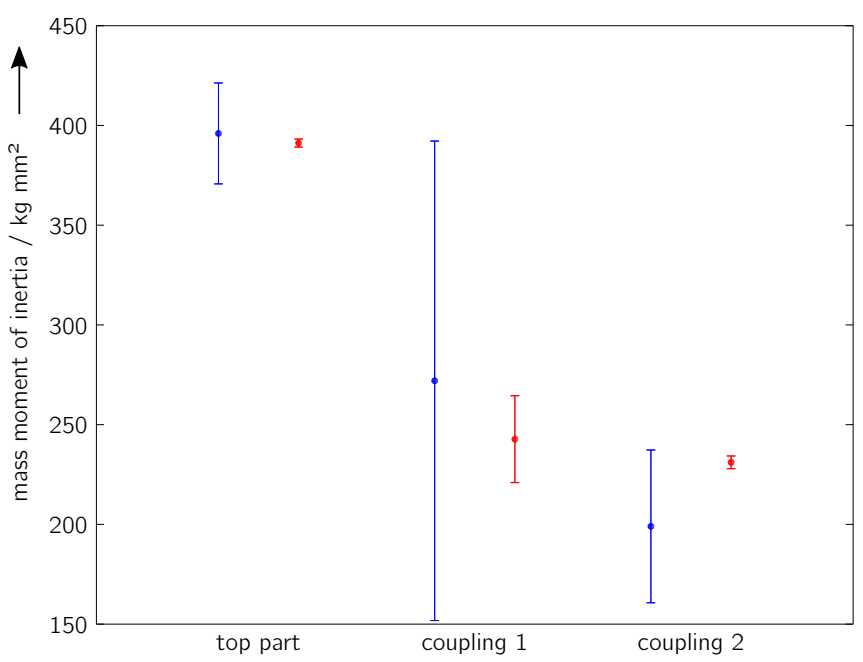

Fig. 13. Measurement results for the mass moment of inertia of three DUTs determined by means of the physical pendulum set-up (blue) and the torsion pendulum set-up (red) including the assigned expanded measurement uncertainties $(k=2)$.

The torsion pendulum results contain no correlated input quantities. Although measurement results of different measurements are subtracted, correlated input quantities were not assumed. Differently to the procedure with the physical pendulum set-up, the torsion spring was removed and remounted for all measurements; the same applies to the mass bodies and the coupling elements on which the mass bodies were mounted.

The measurement uncertainties for the local gravitational acceleration are so small, because the building in which the measurements were carried out has a narrow grid of measured values of $g_{\text {loc }}$ on different floors [23]. The nearest measurement point of $g_{\text {loc }}$ was just a few metres away from the laboratory with the pendulum set-ups.

\subsection{Additional uncertainty contributions}

It is possible that there are additional uncertainty contributions, which cannot be accounted for in the Monte Carlo simulation. These contributions may become obvious from repeatability measurements. For instance, if a remounting alters the properties of the DUT or if there is a (minor) violation of the assumption of rotational symmetry due to manufacturing or bending, these uncertainty contributions have to be taken into account as well. Therefore, repeatability measurements with the DUT mounted at different angle positions were carried out with each of the set-ups for the measurement uncertainty estimation. The standard uncertainty contributions $s$ from the Monte Carlo simulation $s_{\mathrm{MC}}$ and the contributions from repeatability measurements $s_{\text {rep }}$ were added giving

$$
s=\sqrt{s_{\mathrm{MC}}^{2}+s_{\mathrm{rep}}^{2}} .
$$

This approach is very conservative, because the measurement uncertainty contributions already estimated by means of the Monte Carlo simulation might be taken into account a second time in the results of the repeatability measurements. Nevertheless, it was a feasible way to include the experimentally determined contributions.

\section{Measurement Results}

Comparison measurements of the same devices under test were carried out at both measurement set-ups.

\subsection{Devices under test}

Mass moments of inertia of three different DUTs were determined as detailed below:

- The rotor of an air bearing with a radial grating disk

- Two identically constructed coupling elements as depicted in the top left of Figure 1.

For each device under test, several repetitive measurements were carried out. For this purpose, the device under test was dismounted and remounted for the next measurement run.

\subsection{Comparison of results}

Measurement results including the estimated expanded measurement uncertainties (MU) with an expansion factor $k=2$ are shown in Figure 13 and given in detail in Table 3 for the physical pendulum results and in Table 4 for the torsion pendulum results. It becomes obvious from the results that the two measurement set-ups agree very well within the estimated expanded measurement uncertainties. The relative measurement uncertainties of the torsion pendulum are much smaller compared to the results of the physical pendulum.

The high relative measurement uncertainties of the physical pendulum can be explained by the disadvantageous ratio of the mass moment of inertia of the pendulum $\left(J_{0}\right)$ and of the device under test $\left(J_{\text {DUT }}\right)$, which is caused by the mechanical design. The relative uncertainties for $J_{0}$ itself are satisfying. With a different mechanical design or with DUTs with a higher mass moment of inertia, this ratio would decrease and therefore the resulting relative measurement uncertainties would decrease accordingly.

The significantly higher measurement uncertainty of the DUT coupling 1 compared to coupling 2 is due to a minor deformation of coupling 1 , which is bent a little. This partial rotational asymmetry causes higher deviations; much higher deviations are found in the physical pendulum set-up, because the returning torque is affected as it depends on the angle position at which the DUT is mounted. The mounted mass bodies tend to straighten a bent DUT in the torsion pendulum set-up as well.

\section{SUMMARY}

For the determination of the mass moment of inertia of certain components of a dynamic torque calibration device, two measurement set-ups were designed and commissioned. The two set-ups incorporate different measurement principles (a physical pendulum and a torsion pendulum). The measurement 
Table 3. Measurement results for the determination of the mass moment of inertia with the physical pendulum.

\begin{tabular}{lrrr}
\hline DUT & $\begin{array}{r}\text { mass moment of inertia / } \\
\mathrm{kg} \mathrm{mm}^{2}\end{array}$ & $\begin{array}{r}\text { expanded MU } \\
U(k=2) / \mathrm{kg} \mathrm{mm}^{2}\end{array}$ & $\begin{array}{r}\text { relative exp. MU } \\
U_{\text {rel }}(k=2)\end{array}$ \\
\hline top part and pendulum (5 measurements) & $2.25 \times 10^{4}$ & 43.14 & $0.21 \%$ \\
pendulum $\left(J_{0}\right)$ (5 measurements) & $2.21 \times 10^{4}$ & 35.35 & $0.17 \%$ \\
\hline coupling 1 (3 measurements) & 272.38 & 120.18 & $44.12 \%$ \\
coupling 2 (4 measurements) & 198.83 & 38.31 & $19.27 \%$ \\
top part (5 measurements) & 395.60 & 25.31 & $6.4 \%$ \\
\hline
\end{tabular}

Table 4. Measurement results for the determination of the mass moment of inertia with the torsion pendulum.

\begin{tabular}{lrrr}
\hline DUT & $\begin{array}{r}\text { mass moment of inertia / } \\
\mathrm{kg} \mathrm{mm}^{2}\end{array}$ & $\begin{array}{r}\text { expanded MU } \\
U(k=2) / \mathrm{kg} \mathrm{mm}^{2}\end{array}$ & $\begin{array}{r}\text { relative exp. MU } \\
U_{\text {rel }}(k=2)\end{array}$ \\
\hline coupling 1 (20 measurements) & 242.71 & 21.78 & $8.97 \%$ \\
coupling 2 (20 measurements) & 231.11 & 3.19 & $1.38 \%$ \\
top part (20 measurements) & 395.67 & 3.77 & $0.95 \%$ \\
\hline
\end{tabular}

uncertainties for both set-ups were estimated and the results are presented.

The measurements show a good agreement of the two independent measurements. With the given devices under test, the torsion pendulum set-up has smaller measurement uncertainties. The higher uncertainties of the physical pendulum are caused by the mechanical design of the set-up and not by the measurement principle. The relative uncertainties would be significantly smaller for heavier DUTs or with a different design of the set-up.

\section{ACKNOWLEDGEMENT}

The author would like to thank his colleague M. Röhr for designing and manufacturing the torsion spring and carrying out most of the torsion pendulum measurements.

This work was part of the Joint Research Project IND09 Traceable Dynamic Measurement of Mechanical Quantities of the European Metrology Research Programme (EMRP). The EMRP is jointly funded by the EMRP participating countries within EURAMET and the European Union.

\section{REFERENCES}

[1] Genta, G., Delprete, C. (1994). Some considerations on the experimental determination of moments of inertia. Meccanica 29 (2), 125-141. doi: 10.1007/BF01007497.

[2] Soulé, K.A., Miller, M.P. (1933). The experimental determination of the moments of inertia of airplanes. NACA Technical Report 467, National Advisory Committee for Aeronautics, Langley Field, USA.
[3] Gracey, W. (1948). The experimental determination of the moments of inertia of airplanes by a simplified compound-pendulum method. NACA Technical Note 1629, National Advisory Committee for Aeronautics, Langley Field, USA.

[4] Jardin, M., Mueller, E. (2007). Optimized Measurements of UAV Mass Moment of inertia with a bifilar pendulum. In AIAA Guidance, Navigation and Control Conference and Exhibit, 20-23 August 2007, Hilton Head, USA. doi: 10.2514/6.2007-6822.

[5] Swank, A.J. (2012). Precision mass property measurements using a five-wire torsion pendulum. In 27th Annual Meeting of the American Society for Precision Engineering, 21-26 October 2012, San Diego, USA.

[6] Kooijman, J.D.G., Schwab, A.L., Meijaard, J.P. (2008). Experimental validation of a model of an uncontrolled bicycle. Multibody System Dynamics 19 (1-2), 115-132. doi: 10.1007/s11044-007-9050-X.

[7] Pedersen, N.F., Sørensen, O.H. (1977). The compound pendulum in intermediate laboratories and demonstrations. American Journal of Physics 45 (10), 994-998. doi: 10.1119/1.10867.

[8] Robertson, R.L., Harris, E.C. (1962). A simplified technique for precision measurement of moment of inertia. In 21st National Conference of the SAWE, 14-17 May 1962, Seattle, USA. Society of Allied Weight Engineer.

[9] Klaus, L. (2015). Dynamic torque calibration. PTB Mitteilungen 125 (2), 12-17. doi: 10.7795/310.20150203.

[10] Bruns, T., Wedmann, A. (2006). Experimentelle Bestimmung von Massenträgheitsmomenten als Ruck- 
fuhrungsgroße fur die dynamische Drehmomentkalibrierung (Experimental determination of moments of inertia for traceability in dynamic torque calibration). tm - Technisches Messen 73 (12), 692-697. doi: 10.1524/teme.2006.73.12.692.

[11] Klaus, L., Bruns, T., Kobusch, M. (2014). Modelling of a dynamic torque calibration device and determination of model parameters. Acta IMEKO 3 (2), 14-18. doi: 10.21014/acta_imeko.v3i2.79.

[12] Peschel, D., Mauersberger, D. (1994). Determination of the friction of aerostatic radial bearings for the levermass system of torque standard machines. In XIII IMEKO World Congress, 5-9 September 1994, Turin, Italy. IMEKO, 216-220.

[13] Beléndez, A., Pascual, C., Méndez, D.I., Beléndez, T., Neipp, C. (2007). Exact solution for the nonlinear pendulum. Revista Brasileira de Ensino de Física 29 (4), 645-648. doi: 10.1590/S1806-11172007000400024.

[14] Baker, G.L., Blackburn, J.A. (2005). The Pendulum. Oxford University Press.

[15] Ochs, K. (2011). A comprehensive analytical solution of the nonlinear pendulum. European Journal of Physics 32 (2), 479-490. doi: 10.1088/0143-0807/32/2/019.

[16] Abramowitz, M., Stegun, I.A. (eds.) (1964; 1972 - 10th printing with corrections). Handbook of Mathematical Functions with Formulas, Graphs, and Mathematical Tables. United States Department of Commerce.

[17] Symon, K.R. (1960). Mechanics. 2nd edition. Addison-
Wesley.

[18] Endevco Meggit Sensing Systems (2006). Guide to adhesively mounting accelerometers. Technical Report.

[19] Joint Committee for Guides in Meteorology. (2008). Evaluation of measurement data - Guide to the expression of uncertainty in measurement. JCGM 100:2008.

[20] Joint Committee for Guides in Meteorology. (2008). Evaluation of measurement data-- Supplement 1 to the "Guide to the expression of uncertainty in measurement"Propagation of distributions using a Monte Carlo method. JCGM 101:2008.

[21] Wübbeler, G., Harris, P.M., Cox, M.G., Elster, C. (2010). A Two-stage procedure for determining the number of trials in the application of a Monte Carlo method for uncertainty evaluation. Metrologia 47 (3), 317-325. doi: 10.1088/0026-1394/47/3/023.

[22] Free Software Foundation, Inc. GNU Octave. http: //www. octave.org/.

[23] Lindau, A., Kumme, R., Heiker, A. (2002). Investigation in the local gravity field of a force laboratory of PTB. In Joint International Conference IMEKO TC3/TC5/TC20: Proceedings of the International Conference on Force, Mass, Torque, Hardness and Civil Engineering Metrology in the Age Globalization, 24-26 September 2002, Celle, Germany. VDI-Berichte Nr. 1685.

Received October 19, 2016. Accepted January 25, 2017. 